Statistics and Nosé formalism for Ehrenfest dynamics

This content has been downloaded from IOPscience. Please scroll down to see the full text. 2011 J. Phys. A: Math. Theor. 44395004

(http://iopscience.iop.org/1751-8121/44/39/395004)

View the table of contents for this issue, or go to the journal homepage for more

Download details:

IP Address: 155.210.92.116

This content was downloaded on 15/10/2013 at 11:09

Please note that terms and conditions apply. 


\title{
Statistics and Nosé formalism for Ehrenfest dynamics
}

\author{
J L Alonso ${ }^{1,2,3}$, A Castro ${ }^{2}$, J Clemente-Gallardo ${ }^{1,2,3}$, J C Cuchí ${ }^{4}$, \\ P Echenique $^{1,2,3,5}$ and $\mathbf{F}$ Falceto ${ }^{1,2}$ \\ ${ }^{1}$ Departamento de Física Teórica, Universidad de Zaragoza, Campus San Francisco, \\ 50009 Zaragoza, Spain \\ 2 Instituto de Biocomputación y Física de Sistemas Complejos (BIFI), Universidad de Zaragoza, \\ Edificio I+D, Mariano Esquillor s/n, 50018 Zaragoza, Spain \\ ${ }^{3}$ Unidad asociada IQFR-BIFI, Spain \\ ${ }^{4}$ Departament d'Enginyeria Agroforestal, ETSEA- Universitat de Lleida, Av. Alcalde Rovira \\ Roure 191, 25198 Lleida, Spain \\ 5 Instituto de Química Física 'Rocasolano' (CSIC), C/ Serrano 119, 28006 Madrid, Spain \\ E-mail: alonso.buj@gmail.com, acastro@bifi.es, jesus.clementegallardo@bifi.es, \\ cuchi@eagrof.udl.cat, echenique.p@gmail.com and falceto@unizar.es
}

Received 7 April 2011, in final form 26 July 2011

Published 7 September 2011

Online at stacks.iop.org/JPhysA/44/395004

\begin{abstract}
Quantum dynamics (i.e. the Schrödinger equation) and classical dynamics (i.e. Hamilton equations) can both be formulated in equal geometric terms: a Poisson bracket defined on a manifold. In this paper, we first show that the hybrid quantum-classical dynamics prescribed by the Ehrenfest equations can also be formulated within this general framework, what has been used in the literature to construct propagation schemes for Ehrenfest dynamics. Then, the existence of a well-defined Poisson bracket allows us to arrive to a Liouville equation for a statistical ensemble of Ehrenfest systems. The study of a generic toy model shows that the evolution produced by Ehrenfest dynamics is ergodic and therefore the only constants of motion are functions of the Hamiltonian. The emergence of the canonical ensemble characterized by the Boltzmann distribution follows after an appropriate application of the principle of equal a priori probabilities to this case. Once we know the canonical distribution of an Ehrenfest system, it is straightforward to extend the formalism of Nosé (invented to do constant temperature molecular dynamics by a non-stochastic method) to our Ehrenfest formalism. This work also provides the basis for extending stochastic methods to Ehrenfest dynamics.
\end{abstract}

PACS numbers: 31.15.xv, 31.15.xr, 02.70.Ns

\section{Introduction}

The Schrödinger equation for a combined system of electrons and nuclei enables us to predict most of the chemistry and molecular physics that surrounds us, including biophysical processes of great complexity. Unfortunately, this task is not possible in general, 
and approximations need to be made, one of the most important and successful being the classical approximation for a number of the particles. Mixed quantum-classical dynamical (MQCD) models are therefore necessary and widely used.

The so-called Ehrenfest equations (EE) result from a straightforward application of the classical limit to a portion of the particles of a full quantum system, and constitute the most evident MQCD model, as well as a first step in the intricated problem of mixing quantum and classical dynamics. For example, it can be noted that much of the field of molecular dynamics (MD) — whether ab initio or not—is based on equations that can be obtained from further approximations to the Ehrenfest model: for instance, written in the adiabatic basis, the Ehrenfest dynamics collapse into Born-Oppenheimer MD if we assume the non-adiabatic couplings to be negligible.

It is not the purpose of this work, however, to dwell on the justification or validity conditions of the EE (see for example [1-5] for rigourous analyses). Nor is it to investigate the unsettled problem of which is the best manner of mixing quantum and classical degrees of freedom. The Ehrenfest model has a proven application niche, and for this reason we are interested in investigating some of its theoretical foundations, in a manner and with an aim that we describe in the following. For recent progress in non-adiabatic electronic dynamics in MQCD see, for example, [6].

Classical mechanics (CM) can be formulated in several mathematical frameworks, each corresponding to a different level of abstraction (Newton equations, the Hamiltonian formalism, the Poisson brackets, etc). Perhaps its more abstract and general formulation is geometrical, in terms of Poisson manifolds. Similarly, quantum mechanics (QM) can be formulated in different ways, some of which resemble its classical counterpart. For example, the observables (self-adjoint linear operators) are endowed with a Poisson algebra almost equal to the one that characterizes the dynamical variables in CM. Moreover, the Schrödinger equation can be recast into the Hamiltonian equation form (see [7]) by transforming the complex Hilbert space into a real one of double dimension; the observables are also transformed into dynamical functions in this new phase space, in analogy with the classical case. Finally, a Poisson bracket formulation has also been established for QM, which permits us to classify both the classical and the quantum dynamics under the same heading.

This variety of formulations does not emerge from academic caprice; the successive abstractions simplify further developments of the theory, such as the step from microscopic dynamics to statistical dynamics: the derivation of the Liouville equation (or the von Neumann equation in the quantum case), at the heart of statistical dynamics, is based on the properties of the Poisson algebra.

The issue regarding what is the correct equilibrium distribution of a mixed quantumclassical system is seen as a very relevant one [8-16]. An attempt to its derivation can be found in [14], where they arrive at the same distribution that we will advocate below, although it is found using the Nosé-Hoover technique [17, 18], which in principle is only a mathematical scheme to produce the equilibrium, and which the very authors of [14] agree that it is not clear how to apply to a mixed quantum-classical dynamics. On the other hand, in [8] they provide some analytical results about this distribution, but not in the case in which the system of interest is described by the EE; instead (and as in $[9,10]$ ), they assume that the system is fully quantum, and that it is coupled to an infinite classical bath via an Ehrenfest-like interaction.

It is therefore necessary to base Ehrenfest dynamics-or any other MQCD model-on a firm theoretical ground. In particular, we are interested in establishing a clear path to statistical mechanics for Ehrenfest systems, which in our opinion should be done by first embedding this dynamics into the same theoretical framework used in the pure classical or quantum cases (i.e. Poisson brackets, symplectic forms, etc). Then, the study of their statistics will follow the 
usual steps for purely classical or purely quantum ensembles. In this respect, it should be noted that other approaches to MQCD (not based on EE) exist [19-21], and to their corresponding statistics [22, 23], however, it has been found that the formulation of well-defined quantumclassical brackets (i.e. satisfying the Jacobi identity and Leibniz derivation rule) is a difficult issue [24-27]. On the contrary, as shown below, and as a result of the fact that the evolution of a quantum system can be formulated in terms of Hamilton equations similar to those of a classical system $[4,28]$, we will not have difficulties in a rigorous formulation of the Ehrenfest dynamics in terms of Poisson brackets.

The road map of this project is the following. In section 2, we recall the definition of the Ehrenfest model. In section 3, we quickly summarize the formulation of CM in terms of Poisson brackets. Then we summarize, in section 4, the analogous description of QM in terms of geometrical objects which can be found, for example, in [29, 7, 30-36]. These works demonstrate how the Schrödinger equation can be written as a set of (apparently classical) Hamiltonian equations. Also, by using a suitable definition of observables as functions on the (real) set of physical states, the Schrödinger equation can be written in terms of a canonical Poisson bracket. QM appears in this way as 'classical', although the existence of an extra algebraic structure encodes the probabilistic interpretation of measurements, and the superposition and indetermination principles [29-33]. As we have in mind the application to computer simulations, we will consider only finite-dimensional quantum systems, obtained by a suitable sample of the electronic states. This simplifies significantly the description, even if the framework is valid also for the infinite-dimensional case (see [33]).

For the case of Ehrenfest dynamics, we can now combine it (section 5) with CM, whose Poisson bracket formulation is very well known. This is the first of the contributions of the paper: once we realize that the geometric description of classical and quantum dynamics is formally analogous, we can combine them as we do when combining different classical systems. The procedure is very simple: we consider as a global phase space the Cartesian product of the electronic and the nuclear phase spaces and define a global Poisson bracket simply as the sum of the classical and the quantum ones. It is completely straightforward to prove that such a Poisson bracket is well defined and that it provides the correct dynamical equations (EE). From a formal point of view, the resulting dynamics is more similar to a classical system than to a quantum one, although when considering a pure quantum system the dynamics is the usual Schrödinger one. This is not surprising since the coupling of the classical and quantum systems makes the total system nonlinear in its evolution, and this is one of the most remarkable differences between a classical and a quantum system in any formulation.

Once the dynamical description as a Poisson system is at our disposal, it is a very simple task (section 6) to construct the corresponding statistical description following the lines of [37] and [38]. This is another main result of the paper: not having proved the Hamiltonian nature of Ehrenfest dynamics, it was not possible to provide a self-consistent definition of the statistics corresponding to it. Once the Hamiltonian description is available, the definition of this statistics is straightforward.

The formal similarity with the CM case ensures the correctness of the procedure and allows us to derive a Liouville equation. The choice of the equilibrium distribution is based, as usual, on the principle of equal a priori probablities implicitly used by Gibbs and clearly formulated by Tolman [39]. After this, in section 7 we extend Nosé formalism to the Ehrenfest MQCD framework and then provide a method to simulate the canonical ensemble of Ehrenfest systems. This is the final main contribution of the paper.

Please note that for systems where the Born-Oppenheimer approximation is not sufficient, the use of the canonical ensemble of a mixed quantum-classical system such as Ehrenfest's is compulsory, since those systems where the electronic gap is small can be affected by electronic 
temperature effects of the same order. Therefore, a simple mechanism of simulation such as Nosé is very important for practical applications.

Finally, in the conclusions (section 8), we propose to extend, within our formalism, stochastic methods to Ehrenfest dynamics.

\section{The Ehrenfest model}

The EE have the following general form:

$$
\begin{aligned}
& \dot{\vec{R}}_{J}(t)=\left\langle\psi(t)\left|\frac{\partial \hat{H}}{\partial \vec{P}_{J}}(\vec{R}(t), \vec{P}(t), t)\right| \psi(t)\right\rangle, \\
& \dot{\vec{P}}_{J}(t)=-\left\langle\psi(t)\left|\frac{\partial \hat{H}}{\partial \vec{R}_{J}}(\vec{R}(t), \vec{P}(t), t)\right| \psi(t)\right\rangle, \\
& \mathrm{i} \hbar \frac{\mathrm{d}}{\mathrm{d} t}|\psi(t)\rangle=\hat{H}(\vec{R}(t), \vec{P}(t), t)|\psi(t)\rangle,
\end{aligned}
$$

where $(\vec{R}, \vec{P})$ denote collectively the set of canonical position and momenta coordinates of a set of classical particles, whereas $\psi$ is the wavefunction of the quantum part of the system (see [40] for the issue of the Hellmann-Feynman theorem in this context, i.e. whether one should take the derivative inside or outside the expectation value).

This and other MQCD models appear in different contexts; in many situations, the division into quantum and classical particles is made after the electrons have been integrated out, and is used to quantize a few of the nuclear degrees of freedom. However, a very obvious case to use $\mathrm{EE}$ is when we want to treat electrons quantum mechanically, and nuclei classically. In order to fix ideas, let us use this case as an example: the Hamiltonian for the full quantum system is

$$
\begin{aligned}
\hat{H}:= & -\hbar^{2} \sum_{J} \frac{1}{2 M_{J}} \nabla_{J}^{2}-\hbar^{2} \sum_{j} \frac{1}{2} \nabla_{j}^{2}+\frac{1}{4 \pi \epsilon_{0}} \sum_{J<K} \frac{Z_{J} Z_{K}}{\left|\vec{R}_{J}-\vec{R}_{K}\right|} \\
& -\frac{1}{4 \pi \epsilon_{0}} \sum_{j<k} \frac{1}{\left|\vec{r}_{j}-\vec{r}_{k}\right|}-\frac{1}{4 \pi \epsilon_{0}} \sum_{J, j} \frac{Z_{J}}{\left|\vec{R}_{J}-\vec{r}_{j}\right|} \\
= & :-\hbar^{2} \sum_{J} \frac{1}{2 M_{J}} \nabla_{J}^{2}-\hbar^{2} \sum_{j} \frac{1}{2} \nabla_{j}^{2}+V_{n-e}(\vec{r}, \vec{R}) \\
= & :-\hbar^{2} \sum_{J} \frac{1}{2 M_{J}} \nabla_{J}^{2}+H_{e}(\vec{r}, \vec{R}),
\end{aligned}
$$

where all sums must be understood as running over the whole natural set for each index. $M_{J}$ is the mass of the $J$ th nucleus in units of the electron mass, and $Z_{J}$ is the charge of the $J$ th nucleus in units of (minus) the electron charge. Also note that we have defined the nuclei-electron potential $V_{n-e}(\vec{r}, \vec{R})$ and the electronic Hamiltonian $H_{e}(\vec{r}, \vec{R})$ operators.

The EE may then be reached in the following way [1-5]: first, the full wavefunction is split into a product of nuclear and electronic wavefunctions, which leads to the time-dependent self-consistent field model, in which the two subsystems are quantum and coupled. Afterwards, a classical limit procedure is applied to the nuclear subsystem, and the EE emerge naturally. In terms of the nuclei positions $\vec{R}_{J}$ and of the element of the Hilbert space $|\psi\rangle \in \mathcal{H}$ which encodes the state of the electrons of the system, the Ehrenfest dynamics is then given by

$$
\begin{aligned}
& M_{J} \ddot{\overrightarrow{R_{J}}}=-\left\langle\psi\left|\nabla_{J} H_{e}(\vec{r}, \vec{R})\right| \psi\right\rangle, \\
& i \hbar \frac{\mathrm{d}}{\mathrm{d} t}|\psi\rangle=H_{e}(\vec{r}, \vec{R})|\psi\rangle .
\end{aligned}
$$


These equations can be given a Hamiltonian-type description by introducing a Hamiltonian function of the form

$$
H(\vec{R}, \vec{P})=\sum_{J} \frac{\vec{P}_{J}^{2}}{2 M_{J}}+\left\langle\psi\left|H_{e}(\vec{r}, \vec{R})\right| \psi\right\rangle
$$

Then, by fixing a relation of the form $\vec{P}_{J}=M \dot{\vec{R}}_{J}$, we obtain a structure similar to Hamilton equations:

$$
\begin{aligned}
& \dot{\vec{R}}_{J}=\frac{\vec{P}_{J}}{M_{J}}, \\
& \dot{\vec{P}}_{J}=-\left\langle\psi\left|\nabla_{J} H_{e}(\vec{r}, \vec{R})\right| \psi\right\rangle, \\
& i \hbar \frac{\mathrm{d}}{\mathrm{d} t}|\psi\rangle=H_{e}(\vec{r}, \vec{R})|\psi\rangle .
\end{aligned}
$$

This set of equations perhaps constitutes the most evident MQCD model, as well as a first step in the intricated problem of mixing quantum and classical dynamics. In this sense, it is interesting to note that written in the adiabatic basis $\left\{\psi_{m}(\vec{R})\right\}_{m}$ formed by the eigenvectors of the electronic Hamiltonian,

$$
\hat{H}_{\mathrm{e}}(\vec{r}, \vec{R})\left|\psi_{m}(\vec{R})\right\rangle=E_{m}(\vec{R})\left|\psi_{m}(\vec{R})\right\rangle,
$$

the Ehrenfest dynamics collapse into ground state Born-Oppenheimer MD (gsBOMD) if we assume the non-adiabatic couplings to be negligible. Indeed, by expanding the wavefunction in this basis (some relevant considerations related to this change of variables can be found in [40], section 2)

$$
|\psi(t)\rangle=\sum_{m} c_{m}(t)\left|\psi_{m}(\vec{R}(t))\right\rangle
$$

the equations are transformed into

$M_{J} \ddot{\vec{R}}_{J}(t)=-\sum_{m}\left|c_{m}(t)\right|^{2} \nabla_{J} E_{m}(\vec{R}(t))-\sum_{m n} c_{m}^{*}(t) c_{n}(t)\left[E_{m}(\vec{R}(t))-E_{n}(\vec{R}(t))\right] \vec{d}_{J}^{m n}(\vec{R}(t))$,

$\mathrm{i} \frac{\mathrm{d}}{\mathrm{d} t} c_{m}(t)=E_{m}(\vec{R}(t)) c_{m}(t)-\mathrm{i} \sum_{n} c_{n}(t)\left[\sum_{J} \dot{\vec{R}}_{J} \cdot \vec{d}_{J}^{m n}(\vec{R}(t))\right]$

where the 'non-adiabatic couplings' are defined as

$$
\vec{d}_{J}^{m n}(\vec{R}):=\left\langle\psi_{m}(\vec{R}) \mid \nabla_{J} \psi_{n}(\vec{R})\right\rangle .
$$

If these are negligible, and we assume that the electronic system starts from the ground state $\left(c_{m}(0)=\delta_{m 0}\right)$, the EE model reduces to gsBOMD:

$$
\begin{aligned}
& M_{J} \ddot{\vec{R}}_{J}(t)=\nabla_{J} E_{0}(\vec{R}(t)), \\
& c_{m}(t)=\delta_{m 0} .
\end{aligned}
$$

In spite of the formal similarities, the EE equations do not correspond yet to Hamilton equations, since they lack a global phase-space formulation (encompassing both the nuclear and the electronic degrees of freedom) and a Poisson bracket. 


\section{CM in terms of Poisson brackets}

We begin by recalling very quickly the Hamiltonian formulation of classical dynamics (we address the interested reader to a classical text, such as [41], for a detailed presentation). Let us consider a classical system with phase space $M_{C}$, which, for the sake of simplicity, we can identify with $\mathbb{R}^{2 n}$, where $n$ is the number of degrees of freedom (strictly speaking, $M_{C}$ is a general $2 n$-dimensional manifold, homeomorphic to $\mathbb{R}^{2 n}$ only locally). The $2 n$ dimensions correspond with the $n$ position coordinates that specify the configuration of the system, and the $n$ corresponding momenta (mathematically, however, the division into 'position' and 'momenta' coordinates is a consequence of the Darboux theorem-see below).

The 'observables' in CM are differentiable functions

$$
f: M_{C} \rightarrow \mathbb{R}
$$

that assign the result of a measurement to every point in $M_{C}$. On this set of functions $C^{\infty}\left(M_{C}\right)$ we introduce the Poisson bracket, $\{\cdot, \cdot\}$, a bilinear operation

$$
\{\cdot, \cdot\}: C^{\infty}\left(M_{C}\right) \times C^{\infty}\left(M_{C}\right) \rightarrow C^{\infty}\left(M_{C}\right),
$$

which

- is antisymmetric,

$$
\{f, g\}=-\{g, f\}, \quad \forall f, g \in C^{\infty}\left(M_{C}\right),
$$

- satisfies the Jacobi identity, i.e. $\forall f, g, h \in C^{\infty}\left(M_{C}\right)$ :

$$
\{f,\{g, h\}\}+\{h,\{f, g\}\}+\{g,\{h, f\}\}=0,
$$

- and satisfies the Leibniz rule, i.e. $\forall f, g, h \in C^{\infty}\left(M_{C}\right)$ :

$$
\{f, g h\}=\{f, g\} h+g\{f, h\} .
$$

If the Poisson bracket is non-degenerate (it has no Casimir functions), a theorem due to Darboux ensures that there exists a set of coordinates $(\vec{R}, \vec{P})$ for which the bracket has the 'standard' form (at least locally, in a neighbourhood of every point):

$$
\left\{f_{1}, f_{2}\right\}=\sum_{k=1}^{n} \frac{\partial f_{1}}{\partial P_{k}} \frac{\partial f_{2}}{\partial R^{k}}-\frac{\partial f_{1}}{\partial R^{k}} \frac{\partial f_{2}}{\partial P_{k}} .
$$

These coordinates are called Darboux coordinates. They are specially useful when studying the dynamics and invariant measures of a Hamiltonian system [41]. In the rest of the paper, we will work with this kind of coordinates.

The Poisson bracket allows us to introduce the concept of a Hamiltonian vector field: Given a function $f \in C^{\infty}\left(M_{C}\right)$ and a Poisson bracket $\{\cdot, \cdot\}$, a vector field, $X_{f}$, is said to be its Hamiltonian vector field if

$$
X_{f}(g)=\{f, g\}, \quad \forall g \in C^{\infty}\left(M_{C}\right) .
$$

In standard coordinates:

$$
X_{f}=\sum_{k=1}^{n} \frac{\partial f(R, P)}{\partial P_{k}} \frac{\partial}{\partial R^{k}}-\frac{\partial f(R, P)}{\partial R^{k}} \frac{\partial}{\partial P_{k}} .
$$

We will call a Hamiltonian system to a triple $\left(M_{C},\{\cdot, \cdot\}, H\right)$, where $\{\cdot, \cdot\}$ is a Poisson bracket on $M_{C}$, and $H \in C^{\infty}\left(M_{C}\right)$ is the Hamiltonian of the system. The dynamics of a Hamiltonian system can be formulated in two alternative manners: 
- The trajectories of the system are given by the integral curves of the Hamiltonian vector field $X_{H}$.

- If we consider instead the set of classical observables, the dynamics is written as the Poisson bracket of the Hamiltonian function $H$ with any other function, i.e.

$$
\frac{\mathrm{d} f}{\mathrm{~d} t}=\{H, f\}, \quad \forall f \in C^{\infty}\left(M_{C}\right) .
$$

Both approaches are equivalent: the differential equations that determine the integral curves of the Hamiltonian vector field are given by equation (22), for the functions 'position' and 'momenta' of each particle (i.e. $\vec{R}$ and $\vec{P}$ ). These equations are nothing else but Hamilton equations:

$$
\begin{aligned}
& \dot{R}^{j}=\frac{\partial H}{\partial P_{j}}, \\
& \dot{P}_{j}=-\frac{\partial H}{\partial R^{j}} .
\end{aligned}
$$

\section{Summary of geometric QM}

The aim of this section is to provide a description of quantum mechanical systems by using the same geometric tools which are used to describe classical mechanical systems, outlined above. It is just a very quick summary of the framework which has been developed in the last 30 years and which can be found in [29, 7, 30-36] and references therein. For the sake of simplicity, we will focus only on the finite-dimensional case. The Hilbert space $\mathcal{H}$ then becomes isomorphic to $\mathbb{C}^{n}$ for $n$ a natural number, $\mathbb{C}^{n} \sim \mathcal{H}$ :

\subsection{The states}

Consider a basis $\left\{\left|\psi_{k}\right\rangle\right\}$ for $\mathcal{H}$. Each state $|\psi\rangle \in \mathcal{H}$ can be written in that basis with complex components $\left\{z_{k}\right\}$ :

$$
|\psi\rangle=\sum_{k} z_{k}\left|\psi_{k}\right\rangle
$$

We can just take the vector space inherent to the Hilbert space, and turn it into a real vector space $M_{Q}$, by splitting each coordinate in its real and imaginary parts:

$$
z_{k}=q_{k}+\mathrm{i} p_{k} \mapsto\left(q_{k}, p_{k}\right) \in \mathbb{R}^{2 n} \equiv M_{Q} .
$$

We will use real coordinates $\left(q_{k}, p_{k}\right), k=1, \ldots, n$, to represent the points of $\mathcal{H}$ when thought as real manifold elements. From this real point of view the similarities between the quantum dynamics and the classical one described in the previous section will be more evident. Sometimes it will be useful to maintain the complex notation $\psi$ or $z_{k}$ for the elements of the Hilbert space. Please note that, despite the use of ' $q$ ' and ' $p$ ', these coordinates have in principle no relation at all, with the position or the momentum of the quantum system. They are simply the real and imaginary parts of the complex coordinates used to represent the (finite-dimensional) Hilbert space vector in the chosen basis. Therefore, the resulting geometrical description has no relation with the usual phase-space descriptions of QM, such as the Wigner representation, which is defined for infinite-dimensional quantum systems and where the coordinates to represent the point of the phase space do correspond to the spectra of the position and momentum operators. For the same reason, there is no relation with any 
classical limit of the quantum system: the description in terms of these coordinates $(q, p)$ is purely quantum.

There is no one-to-one correspondence between physical states and elements of the Hilbert space used to describe it; it is a well-known fact that physical states are independent of global phases. This ambiguity also translates to the formulation with real vector spaces. Two equivalent states are related by a phase transformation; the best way to characterize these transformations in $M_{Q}$ is by introducing their infinitesimal generator, given by

$$
\Gamma:=\sum_{k}\left(q_{k} \frac{\partial}{\partial p_{k}}-p_{k} \frac{\partial}{\partial q_{k}}\right) .
$$

This is a vector field whose meaning is clear if we realize that a phase change modifies the angle of the complex number representing the state, when considered in polar form (i.e. in polar coordinates $\left\{r_{k}, \theta_{k}\right\}_{k=1, \ldots, n}$ with $z_{k}=r_{k} \mathrm{e}^{\mathrm{i} \theta_{k}}$, equation (25) becomes $\left.\Gamma=\sum_{k} \partial_{\theta_{k}}\right)$. Then, from a geometrical point of view we can use equation (25) in two ways:

- computing its integral curves, which are the different states which are obtained from an initial one by a global phase multiplication;

- acting with the vector field on functions of $M_{Q}$. A function will be acceptable as an observable if it is invariant under phase transformations, i.e. $\Gamma f=0$.

In a quantum Hilbert space, we also consider two states to be equivalent if they merely differ by their norm. One may therefore only consider the sphere of states with norm equal to 1 in $\mathbb{C}^{n}$; this corresponds in the real-vector-space description to the $\left(2 N_{Q}-1\right)$-dimensional sphere:

$$
S_{Q}:=\left\{(\vec{q}, \vec{p}) \in M_{Q} \mid \sum_{k}\left(q_{k}^{2}+p_{k}^{2}\right)=1\right\} .
$$

It is immediate that the vector field $\Gamma$ is tangent to $S_{Q}$, since the phase change preserves the norm of the state.

\subsection{The Poisson bracket}

Taking as coordinates $z_{k}=q_{k}+\mathrm{i} p_{k}$, the components of a vector in an orthonormal basis, we can define a Poisson bracket in $M_{Q}$ by

$$
\{f, g\}:=\sum_{k} \frac{1}{2}\left(\frac{\partial f}{\partial p_{k}} \frac{\partial g}{\partial q_{k}}-\frac{\partial f}{\partial q_{k}} \frac{\partial g}{\partial p_{k}}\right),
$$

that corresponds to the standard Poisson bracket in CM. It will also be useful to introduce a symmetric bracket by

$$
\{f, g\}_{+}:=\sum_{k} \frac{1}{2}\left(\frac{\partial f}{\partial q_{k}} \frac{\partial g}{\partial q_{k}}+\frac{\partial f}{\partial p_{k}} \frac{\partial g}{\partial p_{k}}\right) .
$$

We have made use of a specific basis; one may think that the definition of the brackets could depend on the choice of the basis. However, it is a matter of simple algebra to prove that this is not the case.

An important property of the brackets defined above is that they are preserved by the vector field $\Gamma$ in equation $(25)$ in the sense that

$$
\Gamma\{f, g\}=\{\Gamma f, g\}+\{f, \Gamma g\}
$$

and

$$
\Gamma\{f, g\}_{+}=\{\Gamma f, g\}_{+}+\{f, \Gamma g\}_{+},
$$


a fact that can be proved with a simple computation. This property is important for us because it implies that the symmetric or antisymmetric bracket of two phase-invariant functions will also be phase invariant:

$$
\Gamma f=\Gamma g=0 \Rightarrow \Gamma\{f, g\}=\Gamma\{f, g\}_{+}=0 .
$$

\subsection{The observables}

We now proceed to discuss how to represent the physical observables on this new setting. Instead of considering the observables as linear operators (plus the usual requirements, selfadjointness, boundedness, etc) on the Hilbert space $\mathcal{H}$, we will represent them as functions defined on the real manifold $M_{Q}$, as it is done in CM. But we cannot forget the linearity of the operators, and thus not any function is acceptable. We will consider, for any operator $A \in \operatorname{Lin}(\mathcal{H})$, the quadratic function

$$
f_{A}(\psi):=\langle\psi \mid A \psi\rangle \text {. }
$$

We will denote the set of such functions as $\mathcal{F}_{S}\left(M_{Q}\right)$.

Note that this definition of observable is different from the analogous one in the classical case. In the classical framework, a state, represented by a point in $M_{C}$, provides a well-defined result for any observable $f: M_{C} \rightarrow \mathbb{R}$. In this geometric quantum mechanics (GQM in the following) framework, on the other hand, the value at a given state of $f_{A} \in \mathcal{F}_{S}\left(M_{Q}\right)$ provides just the average value of the corresponding operator in that state. Besides, contrarily to the classical case, not all the $C^{\infty}$ functions on $M_{Q}$ are regarded as observables, but only those of the form of (29).

Once the definition has been stated, one must verify that it is a consistent one, meaning that (i) they are phase-invariant functions $(\Gamma f=0)$, and (ii) the set of observables is closed with respect to both the symmetric and antisymmetric brackets (i.e. the bracket of two elements of $\mathcal{F}_{S}\left(M_{Q}\right)$ is also an element of $\mathcal{F}_{S}\left(M_{Q}\right)$ ). Both facts can be proven by direct computation. The second proof, however, is interesting because it leads us to consider the relevant algebraic operations within $\mathcal{F}_{s}\left(M_{Q}\right)$. In the usual approach of QM, there are three algebraic structures on the set of operators, which turn out to be meaningful and important for the physical description.

- The associative product of two operators:

$$
A, B \in \operatorname{Lin}(\mathcal{H}) \rightarrow A \cdot B \in \operatorname{Lin}(\mathcal{H}) .
$$

It is important to note, though, that this operation is not internal in the set of Hermitian operators (i.e. those associated with physical magnitudes), since the product of two Hermitian operators is not Hermitian, in general.

- The anticommutator of two operators:

$$
A, B \in \operatorname{Lin}(\mathcal{H}) \rightarrow[A, B]_{+} \in \operatorname{Lin}(\mathcal{H}) .
$$

- The commutator of two operators:

$$
A, B \in \operatorname{Lin}(\mathcal{H}) \rightarrow \mathrm{i}[A, B] \in \operatorname{Lin}(\mathcal{H})
$$

Note that these last two operations are internal in the space of Hermitian operators. How do we translate these operations into the GQM scheme? If we take $f_{A}, f_{B} \in \mathcal{F}_{s}\left(M_{Q}\right)$, 
- the anticommutator of two operators becomes the symmetric bracket or Jordan product of the functions (see [42]):

$$
[A, B]_{+} \rightarrow f_{[A, B]_{+}}=\left\{f_{A}, f_{B}\right\}_{+} \in \mathcal{F}_{S}\left(M_{Q}\right),
$$

- the commutator of two operators translates into the Poisson bracket of the functions:

$$
\mathrm{i}[A, B] \rightarrow f_{i[A, B]}=\left\{f_{A}, f_{B}\right\} \in \mathcal{F}_{S}\left(M_{Q}\right) .
$$

We may conclude, therefore, that the operators and their algebraic structures are encoded in the set of functions with the brackets associated with the Hermitian product. Thus, we see why it makes sense to consider only that specific type of functions: it is a choice which guarantees to maintain all the algebraic structures which are required in the quantum description.

Another important property of the set of operators of QM is the corresponding spectral theory. In any quantum system, it is of utmost importance to be able to find eigenvalues and eigenvectors. In GQM, these objects are considered in the following way: let $f_{A}$ be the function associated with the observable $A$. Then, if we consider $\psi \in S_{Q}$,

- the eigenvectors of the operator $A$ coincide with the critical points of the function $f_{A}$, i.e. $\mathrm{d} f_{A}(\psi)=0 \Rightarrow \psi$ is an eigenvector of $A$,

- the eigenvalue of $A$ at the eigenvector $\psi$ is the value that the function $f_{A}$ takes at the critical point $\psi$.

We finalize this discussion on the observables by noting that the identity operator $\mathbb{I}$ corresponds with the function

$$
f_{\mathbb{I}}(q, p)=\sum_{k}\left(q_{k}^{2}+p_{k}^{2}\right),
$$

and the Hamiltonian vector field of this function is precisely the generator of phase transformations:

$$
\left\{f_{\mathbb{I}}, \cdot\right\}=\sum_{k}\left(p_{k} \frac{\partial}{\partial q_{k}}-q_{k} \frac{\partial}{\partial p_{k}}\right)=\Gamma .
$$

\subsection{The dynamics}

As in the classical case, the dynamics can be implemented in different forms, always in a way which is compatible with the geometric structures introduced so far.

- In the Schrödinger picture, the dynamics is described as the integral curves of the vector field $X_{f_{H}}$, where $H$ is the Hamiltonian operator of the system:

$$
X_{f_{H}}=\hbar^{-1}\left\{f_{H}, \cdot\right\} \text {. }
$$

- In the Heisenberg picture, the dynamics is introduced by transferring the Heisenberg equation into the language of functions:

$$
\dot{f}_{A}=\hbar^{-1}\left\{f_{H}, f_{A}\right\} \text {. }
$$

For example, the von Neumann equation is rewritten as

$$
\dot{f}_{\rho}=\hbar^{-1}\left\{f_{H}, f_{\rho}\right\},
$$

where $f_{\rho}$ is the function associated with the density matrix. 
Therefore, it is possible to describe quantum dynamics as a flow of a vector field on the manifold $M_{Q}$ or on the set of quadratic functions of the manifold. Such a vector field is a Hamiltonian vector field with respect to the Poisson bracket encoded in the Hermitian structure of the system.

The dynamics thus defined preserves the norm of the state, which allows us to restrict the set of admissible states to those with norm equal to 1 . This can be proved by analysing the evolution of the function $f_{\mathbb{I}}$, given by the Poisson bracket with the Hamiltonian:

$$
\dot{f}_{\mathbb{I}}=\hbar^{-1}\left\{f_{H}, f_{\mathbb{I}}\right\} \text {. }
$$

But, because of the properties of the bracket, $\left\{f_{H}, f_{\mathbb{I}}\right\}=f_{[H, \mathbb{I}]}=0$, and thus the flow is restricted to the sphere $S_{Q}$.

Example 1. Let us consider a simple two-level quantum system, defined on $\mathbb{C}^{2}$. As a real manifold, $M_{Q} \sim \mathbb{R}^{4}$. Then, consider a Hamiltonian $H: \mathbb{C}^{2} \rightarrow \mathbb{C}^{2}$ :

$$
H=\left[\begin{array}{ll}
H_{11} & H_{12} \\
H_{21} & H_{22}
\end{array}\right] .
$$

If we consider it as a matrix on the real vector space $M_{Q}$, it reads

$$
H_{\mathbb{R}}=\left[\begin{array}{llll}
H_{q_{1} q_{1}} & H_{q_{1} p_{1}} & H_{q_{1} q_{2}} & H_{q_{1} p_{2}} \\
H_{p_{1} q_{1}} & H_{p_{1} p_{1}} & H_{p_{1} q_{2}} & H_{p_{1} p_{2}} \\
H_{q_{2} q_{1}} & H_{q_{2} p_{1}} & H_{q_{2} q_{2}} & H_{q_{2} p_{2}} \\
H_{p_{2} q_{1}} & H_{p_{2} p_{1}} & H_{p_{2} q_{2}} & H_{p_{2} p_{2}}
\end{array}\right] .
$$

It is easy to prove that this matrix is symmetric if the Hamiltonian is Hermitian $\left(H_{12}=H_{21}^{*}\right)$.

The function $f_{H}$ in $\mathcal{F}_{S}\left(M_{Q}\right)$ thus becomes

$$
f_{H}=\psi_{\mathbb{R}}^{t} H_{\mathbb{R}} \psi_{\mathbb{R}}, \quad \text { where } \quad \psi_{\mathbb{R}}=\left(q_{1}, p_{1}, q_{2}, p_{2}\right)^{t}
$$

and then, the Hamiltonian vector field turns out to be

$$
X_{H}=\hbar^{-1}\left(\frac{\partial f_{H}}{\partial p_{1}} \frac{\partial}{\partial q_{1}}-\frac{\partial f_{H}}{\partial q_{1}} \frac{\partial}{\partial p_{1}}+\frac{\partial f_{H}}{\partial p_{2}} \frac{\partial}{\partial q_{2}}-\frac{\partial f_{H}}{\partial q_{2}} \frac{\partial}{\partial p_{2}}\right)
$$

and its integral curves are precisely the expression of the Schrödinger equation when we write it back in complex terms:

$$
\begin{aligned}
& \dot{q}_{1}=\hbar^{-1}\left(H_{p_{1} q_{1}} q_{1}+H_{p_{1} q_{2}} q_{2}+H_{p_{1} p_{1}} p_{1}+H_{p_{1} p_{2}} p_{2}\right), \\
& \dot{p}_{1}=-\hbar^{-1}\left(H_{q_{1} q_{1}} q_{1}+H_{q_{1} q_{2}} q_{2}+H_{q_{1} p_{1}} p_{1}+H_{q_{1} p_{2}} p_{2}\right), \\
& \dot{q}_{2}=\hbar^{-1}\left(H_{p_{2} q_{2}} q_{2}+H_{p_{2} q_{1}} q_{1}+H_{p_{2} p_{2}} p_{2}+H_{p_{2} p_{1}} p_{1}\right), \\
& \dot{p}_{2}=-\hbar^{-1}\left(H_{q_{2} q_{2}} q_{2}+H_{q_{2} q_{1}} q_{1}+H_{q_{2} p_{2}} p_{2}+H_{q_{2} p_{1}} p_{1}\right) .
\end{aligned}
$$

We can write these equations as

$$
\dot{\psi_{\mathbb{R}}}=-\hbar^{-1} \mathbf{J} H_{\mathbb{R}} \psi_{\mathbb{R}}, \quad \text { with } \quad \mathbf{J}=\left[\begin{array}{cccc}
0 & -1 & 0 & 0 \\
1 & 0 & 0 & 0 \\
0 & 0 & 0 & -1 \\
0 & 0 & 1 & 0
\end{array}\right]
$$

or, equivalently,

$$
|\dot{\psi}\rangle=-\hbar^{-1} \mathrm{i} H|\psi\rangle, \quad \text { where } \quad|\psi\rangle=\left[\begin{array}{l}
q_{1}+\mathrm{i} p_{1} \\
q_{2}+\mathrm{i} p_{2}
\end{array}\right]
$$

is the complex state vector in terms of the real coordinates. The operator $\mathbf{J}$, that satisfies $\mathbf{J}^{2}=-I$, is called the complex structure. As it is apparent when comparing (38) and (39), it implements the multiplication by the imaginary unity $i$ in the real presentation of the Hilbert space. Observe that $\mathbf{J}$ and $H_{\mathbb{R}}$ commute, this is due to the fact that the latter comes from an operator $H$ in the complex Hilbert space. 


\section{Ehrenfest dynamics as a Hamiltonian system}

In this section, we show how to put together the dynamics of a quantum and a classical system following the presentation of the two previous sections. Thus, we are describing a physical system characterized by the following elements.

\subsection{The set of states of our system}

- First, let $\mathcal{H}$ be a Hilbert space which describes the quantum degrees of freedom of our system. For example, it could describe the electronic subsystem; in this case, it is the vector space corresponding to the completely antisymmetric representation of the permutation group $S_{N}$ (i.e. a set of Slater determinants), where $N$ is the number of electrons of the system and each electron lives in a Hilbert space of dimension $M$. Thus, the dimension of $\mathcal{H}$ will be $N_{Q}=\frac{M !}{N !(M-N) !}$.

We know that it is a complex vector space, but we prefer to consider it as a real vector space with the double of degrees of freedom and denote it as $M_{Q}$. Also, in correspondence with the Hilbert space vectors in the usual formalism of QM, several states in $M_{Q}$ represent the same physical state. To consider true physical states one should extract only those corresponding to the projective space, which can be identified with a submanifold of $M_{Q}$. A more general approach is to consider the sphere of states with norm equal to $1, S_{Q}$, and take into account the phase transformations generated by equation (25) in a proper way. We will discuss this in the following sections.

- Second, let $M_{C}$ be a differentiable manifold which contains the classical degrees of freedom. We will assume it to be a phase space, and thus it will have an even number of degrees of freedom and it will be endowed with a non-degenerate Poisson bracket that in Darboux cordinates $(\vec{R}, \vec{P})$ reads

$$
\{f, g\}_{C}=\sum_{J} \frac{\partial f}{\partial P_{J}} \frac{\partial g}{\partial R_{J}}-\frac{\partial f}{\partial R_{J}} \frac{\partial g}{\partial P_{J}} .
$$

- Third, we let our state space $\mathcal{S}$ be the Cartesian product of both manifolds:

$$
\mathcal{S}:=M_{C} \times M_{Q}
$$

Such a description has important implications: it is possible to consider each subsystem separately in a proper way but it is not possible to entangle the subsystems with one another. As long as Ehrenfest dynamics disregards this possibility, the choice of the Cartesian product is the most natural one.

The coordinates that describe the state of the system are as follows.

- The positions and momenta of the nuclei:

$$
(\vec{R}, \vec{P}) \in M_{C} \text {. }
$$

We will have $3 N_{C}+3 N_{C}$ of these, for $N_{C}$ the number of nuclei of the system.

- The real and imaginary parts of the coordinates of the Hilbert space vectors with respect to some basis:

$$
(\vec{q}, \vec{p}) \in M_{Q} .
$$

We will have $N_{Q}+N_{Q}$ of these, for $N_{Q}$ the complex dimension of the Hilbert space $\mathcal{H}$. 


\subsection{The observables}

Our observables are functions defined on the state space $S=M_{C} \times M_{Q}$. We know from our discussion in the case of a purely quantum system that any function of the form (29) produces an evolution, via the Poisson bracket, which preserves the norm. In the MQCD case, we can easily write the analogue of the vector field (25) by writing

$$
\Gamma_{Q}=\mathbb{I} \otimes \Gamma \text {. }
$$

This is again the infinitesimal generator of phase transformations for the quantum subsystem, but written at the level of the global state space $M_{C} \times M_{Q}$. A reasonable property to be asked to the functions chosen to represent our observables is to be constant under this transformation. From a mathematical point of view, we will define the set of possible observables, $\mathcal{O}$, as the set of all $C^{\infty}$-functions on the set $M_{C} \times M_{Q}$ which are constant under phase changes on the quantum degrees of freedom, i.e.

$$
\mathcal{O}:=\left\{f \in C^{\infty}\left(M_{C} \times M_{Q}\right) \mid \Gamma_{Q} f=0\right\} .
$$

As we will see later, this choice reflects the fact that, when considered coupled together, the nonlinearity of CM expands also to MQCD. There are some important subsets that should be considered.

- The set of classical functions: these are functions which depend only on the classical degrees of freedom. Mathematically, they can be written as those functions $f \in \mathcal{O}$ such that there exists a function $f_{C} \in C^{\infty}\left(M_{C}\right)$ such that

$$
f(\vec{R}, \vec{P}, \vec{q}, \vec{p})=f_{C}(\vec{R}, \vec{P}) .
$$

We denote this subset as $\mathcal{O}_{C}$. An example of a function belonging to this set is the linear momentum of the nuclei.

- The set of generalized quantum functions: functions which depend only on the quantum degrees of freedom and which are constant under changes in the global phase. Mathematically, they can be written as those functions $f \in \mathcal{O}$ such that there exists a function $f_{Q} \in C^{\infty}\left(M_{Q}\right)$ such that

$$
f(\vec{R}, \vec{P}, \vec{q}, \vec{p})=f_{Q}(\vec{q}, \vec{p}) ; \quad \Gamma\left(f_{Q}\right)=0 .
$$

We denote these functions as $\mathcal{O}_{Q}$. We have added the adjective 'generalized' because this set is too large to represent only the set of pure quantum observables. These later functions should be considered, when necessary, as a smaller subset, which corresponds to the set of functions defined in equation (29). We denote this smaller subset as $\mathcal{O}_{Q}^{s}$. An example of a function belonging to $\mathcal{O}_{Q}^{s}$ is the linear momentum of the electrons.

- A third interesting subset is the set of arbitrary linear combinations of the subsets above, i.e. those functions which are written as the sum of a purely classical function and a purely quantum one:

$$
f(\vec{R}, \vec{P}, \vec{q}, \vec{p})=f_{C}(\vec{R}, \vec{P})+f_{Q}(\vec{q}, \vec{p}) .
$$

We will denote this set as $\mathcal{O}_{C+Q}$. An element of this set of functions is the total linear momentum of the composed system.

We would like to make a final but very important remark. We have not chosen the set of observables as

$$
\mathcal{P}:=\left\{f_{A} \in C^{\infty}\left(M_{C} \times M_{Q}\right) \mid f_{A}=\langle\psi \mid A(\vec{R}, \vec{P}) \psi\rangle\right\},
$$

for $A(\vec{R}, \vec{P})$ a linear operator on the Hilbert space $\mathcal{H}$ depending on the classical degrees of freedom, because of two reasons. 
- It is evident that the set above is a subset of (43) and thus we are not loosing any of these observables. But it is a well-known property that Ehrenfest dynamics is not linear and it does not preserve $\mathcal{P}$. We must thus enlarge the set (46).

- We are going to introduce in the next section a Poisson bracket on the space of observables. For that bracket to close a Poisson algebra, we need to consider the whole set (43).

It is important to note that in the set (43) there are operators which are not representing linear operators for the quantum part of the system and hence the set of properties listed in section 4 for the pure quantum case are meaningless for them. But this is a natural feature of the dynamics we are considering, because of its nonlinear nature.

\subsection{Geometry and the Poisson bracket on the classical-quantum world}

As we assume that both the classical and the quantum subsystems are endowed with Poisson brackets, we face the same problem we have when combining, from a CM perspective, two classical systems. Therefore, it is immediate to conclude that the corresponding Poisson structures can be combined as

$$
\{\cdot, \cdot\}:=\{\cdot, \cdot\}_{C}+\hbar^{-1}\{\cdot, \cdot\}_{Q}
$$

where the term $\{\cdot, \cdot\}_{C}$ acts on the degrees of freedom of the first manifold and $\{\cdot, \cdot\}_{Q}$ acts on the degrees of freedom of the second one. It is a known fact [41] that such a superposition of Poisson brackets always produces a well-defined Poisson structure in the product space.

The set of pure classical functions $\mathcal{O}_{C}$ and the set of quantum generalized functions $\mathcal{O}_{Q}$ are closed under the Poisson bracket. The same happens with the quantum functions $\mathcal{O}_{Q}^{s}$ and the set of linear combinations $\mathcal{O}_{C+Q}$. In mathematical terms, what we have is a family of Poisson subalgebras. This property ensures that the description of purely classical or purely quantum systems, or even both systems at once but uncoupled to each other, can be made within the formalism.

Once the Poisson bracket on $M_{C} \times M_{Q}$ has been introduced, one can prove, in analogy to the full quantum case, that the infinitesimal generator of the phase transformations is the Hamiltonian vector field of the identity operator, $\Gamma_{Q}=\left\{f_{\mathbb{I}}, \cdot\right\}$. Therefore, a function is a legitimate observable if it commutes with $f_{\mathbb{I}}:\left\{f_{\mathbb{I}}, f\right\}=0$.

\subsection{The definition of the dynamics}

Analogously to the description given in sections 3 and 4, the dynamics of the mixed quantum classical systems can be implemented on

- the manifold which represents the set of states by defining a vector field whose integral curves represent the solutions of the dynamics (Schrödinger picture),

- the set of functions (please note the differences between the classical and the quantum cases) defined on the set of states which represent the set of observables of the system. In this case the Poisson bracket of the functions with the Hamiltonian of the system defines the corresponding evolution (Heisenberg picture).

Both approaches are not disconnected, since they can be easily related:

$$
X_{H}=\left\{f_{H}, \cdot\right\},
$$

where we denote by $X_{H}$ the vector field which represents the dynamics on the phase space and by $f_{H}$ the function which corresponds to the Hamiltonian of the complete system.

We can now proceed to our first goal: to provide a Hamiltonian description of Ehrenfest dynamics in terms of a Poisson structure. We thus define the following Hamiltonian system: 
- A state space corresponding to the Cartesian product $M_{C} \times M_{Q}$.

- A set of operators corresponding to the set of functions $\mathcal{O}$ defined in equation (43).

- The Poisson bracket defined in equation (47).

- And finally, the dynamics introduced by the following Hamiltonian function:

$$
f_{H}(\vec{R}, \vec{P}, \vec{q}, \vec{p}):=\sum_{J} \frac{\vec{P}_{J}^{2}}{2 M_{J}}+\left\langle\psi(\vec{q}, \vec{p}) \mid H_{e}(\vec{R}) \psi(\vec{q}, \vec{p})\right\rangle,
$$

where $H_{e}$ is the expression of the electronic Hamiltonian, $M_{J}$ are the masses of the classical subsystem of the nuclei and $\psi(\vec{q}, \vec{p})$ is the real-space representation of the state $\psi$ analogous to $|\psi\rangle$ in equation (39).

As a result, the dynamics of both subsystems are obtained easily. In the Schrödinger picture we obtain

$$
\begin{aligned}
& \dot{\vec{R}}=\frac{\partial f_{H}}{\partial \vec{P}}=M^{-1} \vec{P}, \\
& \dot{\vec{P}}=-\frac{\partial f_{H}}{\partial \vec{R}}=-\operatorname{grad}\left(\left\langle\psi(\vec{q}, \vec{p}) \mid H_{e}(\vec{R}) \psi(\vec{q}, \vec{p})\right\rangle\right), \\
& \dot{q}_{k}=\hbar^{-1} \frac{\partial f_{H}}{\partial p_{k}}, \quad k=1, \ldots, N_{Q}, \\
& \dot{p}_{k}=-\hbar^{-1} \frac{\partial f_{H}}{\partial q_{k}}, \quad k=1, \ldots, N_{Q} .
\end{aligned}
$$

This set of equations corresponds exactly with Ehrenfest dynamics.

Finally, it is important to verify that the dynamics preserves the set of observables $\mathcal{O}$, i.e. for any observable $f,\left\{f_{H}, f\right\} \in \mathcal{O}$. This can be easily proven since we have established that an observable belongs to $\mathcal{O}$ if it Poisson-commutes with $f_{\mathbb{I}}$. Thus, as $f_{H} \in \mathcal{O}$, if we consider an observable $f \in \mathcal{O}$, by the Jacobi identity

$$
\left\{f_{\mathbb{I}},\left\{f_{H}, f\right\}\right\}=-\left\{f,\left\{f_{\mathbb{I}}, f_{H}\right\}\right\}-\left\{f_{H},\left\{f, f_{\mathbb{I}}\right\}\right\}=0 .
$$

\section{A phase-space description of the statistics of the Ehrenfest dynamics}

The next step in our work is the definition of a statistical system associated with the dynamics we introduced above. The first ingredient for that is the definition of the distributions we will describe the system with.

The main conclusion from the previous section is that Ehrenfest dynamics can be described as a Hamiltonian system on a Poisson manifold. Therefore, we are in a situation similar to a standard classical system. We have seen that the dynamics preserves the submanifold $M_{C} \times S_{Q}$ ( $S_{Q}$ being the sphere in equation (26)), and thus it is natural to consider such a manifold as the space of our statistical system.

Thus, we have to construct now a statistical system composed of two subsystems. We may think of introducing a total distribution factorizing as the product of a classical and a quantum distribution. But as the events of the classical and the quantum regimes are not independent, the two probabilities must be combined and not defined through two factorizing functions.

Hence, we will consider a density $F_{Q C} \in \mathcal{O}$ and the canonical volume element $\mathrm{d} \mu_{Q C}$ in the quantum-classical phase space, restricted to $M_{C} \times S_{Q}$ :

$$
\mathrm{d} \mu_{Q C}:=\mathrm{d} \mu_{C} \mathrm{~d} \Omega_{Q}
$$


which allows us to define the macroscopic average for any observable $M \in \mathcal{O}$ :

$$
\langle M\rangle:=\int_{M_{C} \times S_{Q}} M(\vec{R}, \vec{P}, \vec{q}, \vec{p}) F_{Q C}(\vec{R}, \vec{P}, \vec{q}, \vec{p}) \mathrm{d} \mu_{Q C} .
$$

In the rest of the section we will discuss in more detail the ingredients of this definition.

We start by the volume form. Given the classical phase space $M_{C}$ with Darboux coordinates $(\vec{R}, \vec{P})$ (they always exist locally [41]) we define the volume element in the classical phase space by

$$
\mathrm{d} \mu_{C}:=\prod_{J} \mathrm{~d} P_{J} \mathrm{~d} R_{J}
$$

which, as it is well known, is invariant under any purely classical Hamiltonian evolution, and does not depend on the choice of coordinates.

We might proceed in the same way for the quantum part of our system. Indeed, as we have written the quantum dynamics like a classical Hamiltonian one we could use the canonical invariant volume element, described in the previous paragraph, $\mathrm{d} \mu_{Q}$ on $M_{Q}$. However, new complications appear in this case as in the quantum part of the phase space we restrict the integration to $S_{Q}$ and then we look for an invariant volume form in the unit sphere, rather than in the full Hilbert space.

The new ingredient that makes the restriction possible is the fact that all our observables and of course, also the Hamiltonian, are killed by $\Gamma$. This implies, as discussed before, that $f_{\mathbb{I}}$ is a constant of motion. Using this we decompose

$$
\mathrm{d} \mu_{Q}=\mathrm{d} f_{\mathbb{I}} \mathrm{d} \widetilde{\Omega}_{Q},
$$

which is possible on $\mathcal{H} \backslash\{0\}$. The decomposition is not unique, but the restriction (pullback) of $\mathrm{d} \widetilde{\Omega}_{Q}$ to the unit sphere gives a uniquely defined, invariant volume form on $S_{Q}$ that we will denote by $\mathrm{d} \Omega_{Q}$. The decomposition is nothing but the factorization into the radial part and the solid angle volume element.

Example 2. For the simple case we studied above, where $\mathcal{H}=\mathbb{C}^{2}$ and hence $M_{Q}=\mathbb{R}^{4}$ and $S_{Q}$ is a three-dimensional sphere, the volume element above would be

$\mathrm{d} \Omega_{Q}=q_{1} \mathrm{~d} q_{2} \mathrm{~d} p_{1} \mathrm{~d} p_{2}-q_{2} \mathrm{~d} q_{1} \mathrm{~d} p_{1} \mathrm{~d} p_{2}+p_{1} \mathrm{~d} q_{1} \mathrm{~d} q_{2} \mathrm{~d} p_{2}-p_{2} \mathrm{~d} q_{1} \mathrm{~d} q_{2} \mathrm{~d} p_{1}$.

Now, we finally put together the two ingredients to obtain the invariant volume element $\mathrm{d} \mu_{Q C}$ in equation (55).

Now, we discuss the properties we must require from $F_{Q C}$ in order for equation (56) to correctly define the statistical mechanics for the Ehrenfest dynamics.

- The expected value of the constant observable should be that constant which implies that the integral on the whole set of states is equal to 1 :

$$
\int_{M_{C} \times S_{Q}} F_{Q C} \mathrm{~d} \mu_{Q C}=1
$$

- The average for any observable $f_{A}$ of the form (46) associated with a positive definite Hermitian operator $A$ should be positive. This implies the usual requirements of positive probability density in purely classical statistical mechanics.

It is simple to go from this phase-space description to the formulation of a density operator $\rho$ for the quantum system and make the construction closer to the usual description of quantum statistical mechanics. The density operator is defined as follows:

$$
\rho(\vec{R}, \vec{P}):=\int_{S_{Q}} \mathrm{~d} \mu_{Q}(\vec{q}, \vec{p}) F_{Q C}(\vec{R}, \vec{P}, \vec{q}, \vec{p})|\psi(\vec{q}, \vec{p})\rangle\langle\psi(\vec{q}, \vec{p})|,
$$


where $|\psi(\vec{q}, \vec{p})\rangle\langle\psi(\vec{q}, \vec{p})|$ is the projector on the quantum state parametrized by the pair $(\vec{q}, \vec{p})$. The average value of a pure quantum magnitude $A$, represented by the quadratic function $f_{A}$, can be computed as

$$
\langle A\rangle(\vec{R}, \vec{P}):=\operatorname{Tr}(\rho(\vec{R}, \vec{P}) A)=\int_{S_{Q}} \mathrm{~d} \mu_{Q}(\vec{q}, \vec{p}) F_{Q C}(\vec{R}, \vec{P}, \vec{q}, \vec{p}) f_{A}(\vec{q}, \vec{p}) .
$$

To obtain the corresponding total average, it is required to integrate the resulting object on the classical phase space:

$$
\langle A\rangle:=\int_{M_{C}} \mathrm{~d} \mu_{C}(\vec{R}, \vec{P})\langle A\rangle(\vec{R}, \vec{P}) .
$$

So far we have discussed general considerations about the statistical mechanics of our system. Now we should include the dynamics in the game. Given that we have formulated the Ehrenfest dynamics as a Hamiltonian system, it is well known [37] which is the evolution of the density function $F_{Q C}$. It is given by the Liouville equation

$$
\frac{\mathrm{d} F_{Q C}}{\mathrm{~d} t}+\left\{f_{H}, F_{Q C}\right\}=0
$$

where, in the derivation of the equation, it is an essential requirement that the invariance of the volume form under the evolution of the system.

The equilibrium statistical mechanics is obtained by requiring $\dot{F}_{Q C}=0$, which making use of (64) is equivalent to

$$
\left\{f_{H}, F_{Q C}\right\}=0
$$

or, in other words, $F_{Q C}$ should be a constant of motion. An obvious non-trivial constant of motion is given by any function of $f_{H}$. The question is if there are more. To answer this we should examine closely our particular dynamics. In several occasions we have mentioned the nonlinear character of a generic Ehrenfest system. This is an essential ingredient, because due to this fact we do not expect any other constant of motion than a function of the Hamiltonian itself. This is in contrast with the case of linear equations of motion, where the system is necessarily integrable.

The question of the existence of more constants of motion and, therefore, more candidates for the equilibrium density $F_{Q C}$ can be elucidated with a simple example that we discuss below. In it we show the presence of ergodic regions (open regions in the leave of constant energy densely covered by a single orbit). This rules out the existence of constants of motion different from the constant function in the region (i.e. functions of $f_{H}$ ).

Example 3. In the following example we will study a simple toy model in which the coupling between classical and quantum degrees of freedom gives rise to chaotic behaviour and the appearance of ergodic regions.

The system consists of a complex two-dimensional Hilbert space $M_{Q}=\mathbb{C}^{2}$ and a classical $2 \mathrm{D}$ phase space where we define a $1 \mathrm{D}$ harmonic oscillator. Using coordinates $\left(I_{\theta}, \theta\right)$ for the classical variables (action-angle coordinates for the oscillator) and $\Psi \in \mathbb{C}^{2}$ we define the following Hamiltonian:

$$
f_{H}=I_{\theta}+\frac{1}{2}\left\langle\Psi\left|\sigma_{z}+\epsilon \cos (\theta) \sigma_{x}\right| \Psi\right\rangle
$$

with $\sigma_{x}, \sigma_{z}$ the Pauli sigma matrices.

We parametrize the normalized quantum state by

$$
|\Psi\rangle=\mathrm{e}^{\mathrm{i} \alpha}\left[\begin{array}{c}
I_{\phi} \\
\mathrm{e}^{\mathrm{i} \phi} \sqrt{1-I_{\phi}^{2}}
\end{array}\right] .
$$




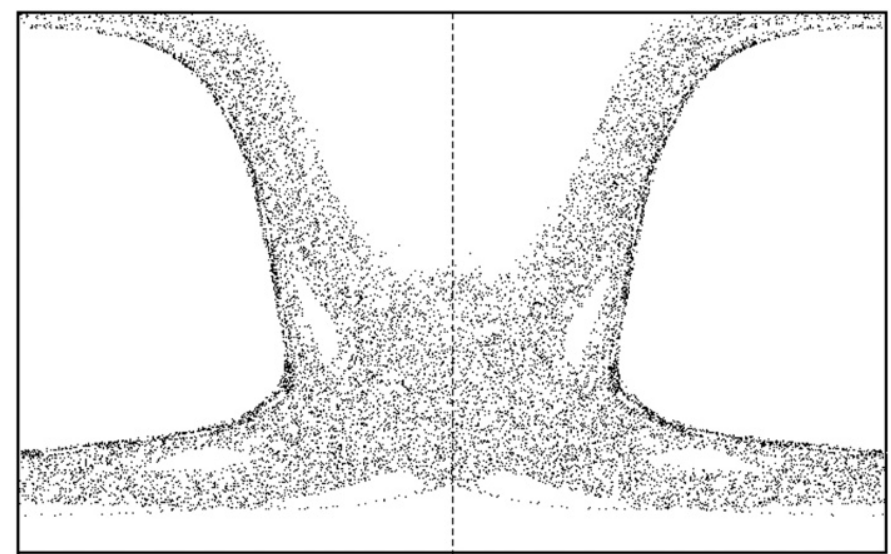

Figure 1. The plot shows a single orbit of the Poincaré map of equation (66) at $\theta=0$. The angle $\phi \in[-\pi, \pi]$ is plotted in the horizontal axis and $I_{\phi} \in[0,1]$ in the vertical one. We have taken $\epsilon=0.8$. The energy determines $I_{\theta}$ and its actual value does not affect the dynamics of $\phi$ and $I_{\phi}$.

One can now easily check that $I_{\phi}$ and $\phi$ are canonical conjugate variables. In these variables the Hamiltonian reads

$$
f_{H}=I_{\theta}+I_{\phi}^{2}+\epsilon I_{\phi} \sqrt{1-I_{\phi}^{2}} \cos (\theta) \cos (\phi),
$$

where $\epsilon$ measures the coupling of the classical and quantum systems.

In the limit of vanishing $\epsilon$ the system is integrable and actually linear in these coordinates. However, for non-vanishing $\epsilon$ the model becomes nonlinear and, as we will show below, regions of chaotic motion emerge.

In order to understand the behaviour of our system it is useful to study its Poincaré map (see [41] for the definition). To this end we take the transversal (or Poincaré) section at $\theta=0$ and, taking into account the conservation of energy, only two coordinates are needed to describe the map, we have chosen the quantum variables $I_{\phi}$ and $\phi .^{6}$

By examining the plot of figure 1 , one sees that the orbit densely fills a large region of the Poincaré section and therefore any constant of motion should be the constant function in that region. In the presence of such an ergodic evolution the only constants of motion are functions of the Hamiltonian.

As a consequence we can claim that, generically within the Ehrenfest dynamics, the only functions which commute with the Hamiltonian in (64) are those which are function of the Hamiltonian $f_{H}$ itself. As the Poisson bracket is skew-symmetric, the property follows trivially. Ergodicity helps us to assert, roughly speaking, that in general no other function in $\mathcal{O}$ will be a constant of the motion.

Thus, at this stage, we can finish our construction with the equilibrium distribution associated with Ehrenfest dynamics. Taking into account the ergodicity of the dynamics, we can impose the equal-probabilities condition to the configurations of an isolated Ehrenfest system, which leads to the microcanonical ensemble (see [43]).

6 In our construction of the Poincaré map we represent the value of $\phi$ and $I_{\phi}$ whenever the periodic coordinate $\theta$ takes the value $\theta=0$. The successive values of $\phi$ and $I_{\phi}$, for some initial condition, form our orbit. As stressed in [41], the Poincaré map is a very useful tool in the context of nonlinear dynamics as many properties of the full system like ergodicity, chaos or regular behaviour, may be inferred from the same property of the Poincaré map. 
In the case in which this system is weakly coupled to a bath, if we assume that the dynamics of the system plus bath is ergodic as well, such that the system plus bath are microcanonically distributed, then the statistics of the Ehrenfest system are given by the well-known canonical probability density (see, for example, [37, 38, 44])

$$
F_{Q C}^{c}(\vec{R}, \vec{P}, \vec{q}, \vec{p})=Z^{-1} \mathrm{e}^{-\beta f_{H}}, \quad Z=\int_{M_{C} \times S_{Q}} \mathrm{e}^{-\beta f_{H}} \mathrm{~d} \mu_{Q C},
$$

where $\beta^{-1}$, proportional to the temperature of the subsystem, is the parameter governing the equilibrium between the system and the bath. An information-theoretic approach to the equiprobability in the microcanonical ensemble and to equation (67) may be seen in [45] or in $[46,47]$ for a much more modern and mathematically sound exposition. We also strongly recommend the recent work of Reimann [48, 49] for a foundation of statistical mechanics under experimentally realistic conditions.

\section{Application: Nosé formalism for Ehrenfest systems}

We now proceed to extend the non-stochastic method proposed by Nosé $[17,18]$ to our Hamiltonian (49). Consider a system defined on $M_{C} \times M_{Q}$ as before and an extension of that system including two new classical degrees of freedom $\left(s, p_{s}\right)$, which produces a total state space

$$
M_{C} \times M_{Q} \times \mathbb{R}^{2}
$$

In this case, in order to include the quantum degrees of freedom, we must take into account the problem of the deformation of the domain (see the change of coordinates of equation (77)) of integration we dealt with in the previous section. Thus, we must rewrite the Hamiltonian given in equation (49) in order to extend the domain of the quantum part from the sphere to the whole $M_{Q}$. In order to do this, we consider

$$
\begin{aligned}
H(\vec{R}, \vec{P}, \vec{q}, \vec{p}) & :=\sum_{k} \frac{\vec{P}_{k}^{2}}{2 M}+\frac{\left\langle\psi(\vec{q}, \vec{p}) \mid H^{e}(\vec{R}) \psi(\vec{q}, \vec{p})\right\rangle}{\langle\psi(\vec{q}, \vec{p}) \mid \psi(\vec{q}, \vec{p})\rangle} \\
& =: H^{N}(\vec{P})+e_{H^{e}}(\vec{q}, \vec{p}, \vec{R}),
\end{aligned}
$$

Now we transform this Hamiltonian into the 'extended' one by introducing a dependence with respect to the new degrees of freedom:

$$
H\left(\vec{R}, \vec{P}, \vec{q}, \vec{p}, s, p_{s}\right):=H^{N}(\vec{P} / s)+e_{H^{e}}(\vec{q}, \vec{p} / s, \vec{R})+\frac{p_{s}^{2}}{2 Q}+g k T \log (s) .
$$

This is very similar to the method proposed by Nosé $[17,18]$ for thermostating classical systems. The Nosé technique is basically an algorithm to produce the correct equilibrium distribution and, therefore, the interpretation of the coordinate $s$ as describing the behaviour of some 'thermostat' is only metaphorical. In the same sense, the particular form of the last two terms in the above Hamiltonian is just the one needed for the theorem that we mention in the following lines to work.

The extended equations of motion now read

$$
\begin{aligned}
& \dot{\vec{R}}=\vec{P} /\left(M s^{2}\right), \\
& \dot{\vec{P}}=-\operatorname{grad}_{\vec{R}}\left(e_{H^{e}}(\vec{q}, \vec{p} / s, \vec{R})\right), \\
& \dot{q}_{k}=\frac{\hbar^{-1} s^{-1}}{\langle\psi \mid \psi\rangle}\left(H_{p_{k} q_{1}} q_{1}+\cdots+H_{p_{k} p_{N_{Q}}} p_{N_{Q}} / s-e_{H^{e}}(\vec{q}, \vec{p} / s, \vec{R}) p_{k}\right),
\end{aligned}
$$




$$
\begin{aligned}
& \dot{p}_{k}=-\frac{\hbar^{-1}}{\langle\psi \mid \psi\rangle}\left(H_{q_{k} q_{1}} q_{1}+\cdots+H_{q_{k} p_{N_{Q}}} p_{N_{Q}} / s-e_{H^{e}}(\vec{q}, \vec{p} / s, \vec{R}) q_{k}\right), \\
& \dot{s}=\frac{p_{s}}{Q} \\
& \dot{p}_{s}=s^{-1} \sum_{k} \dot{q}^{k}+\sum_{j} \dot{R}^{j}-s^{-1} g K T
\end{aligned}
$$

where $k=1, \ldots, N_{Q}$.

Now we generalize the standard result of Nosé $[17,18]$ by proving the equivalence of the microcanonical distribution of the extended system and the canonical distribution of the original one. Equations (70)-(75) are the necessary ones to implement the microcanonical scheme once we assume the ergodic hypothesis for the extended system. To prove the equivalence with the canonical distribution of the original system, we take the Hamiltonian above, and we consider the corresponding microcanonical distribution:

$$
Z=\int_{M_{C} \times M_{Q} \times \mathbb{R}^{2}} \mathrm{~d} \mu_{C} \mathrm{~d} \mu_{Q} \mathrm{~d} p_{s} \mathrm{~d} s \delta\left(H\left(\vec{R}, \vec{P}, \vec{q}, \vec{p}, s, p_{s}\right)-E\right),
$$

where $\mathrm{d} \mu_{C} \mathrm{~d} \mu_{Q}$ is the volume element on $M_{C} \times M_{Q}$.

If we consider the following noncanonical transformation on $M_{C} \times M_{Q} \times \mathbb{R}^{2}$ :

$$
R^{\prime j}=R^{j}, \quad P_{j}^{\prime}=\frac{P_{j}}{s}, \quad q^{\prime k}=q^{k}, \quad p_{k}^{\prime}=\frac{p_{k}}{s}, \quad s^{\prime}=s, \quad p_{s}^{\prime}=p_{s},
$$

the partition function reads (we use the same symbol again for the transformed variables but include the Jacobian which is equal to $s^{D}$ )

$$
\begin{array}{rl}
Z=\int_{M_{C} \times M_{Q} \times \mathbb{R}^{2}} & \mathrm{~d} \mu_{C} \mathrm{~d} \mu_{Q} \mathrm{~d} p_{S} \mathrm{~d} s s^{D} \\
& \times \delta\left(H^{N}(\vec{P})+e_{H^{e}}(\vec{q}, \vec{p}, \vec{R})+\frac{p_{s}^{2}}{2 Q}+g k T \log (s)-E\right),
\end{array}
$$

where $D$ is the number of degrees of freedom of the system (the sum of classical and quantum degrees of freedom).

As the original integral domain includes the complete $M_{Q}$, and therefore a rescaling of momenta does not modify it, we can use the equivalence

$$
\delta(f(s))=\delta\left(s-s_{0}\right) /\left|f^{\prime}\left(s_{0}\right)\right|,
$$

where

$$
s_{0}=\exp \left(-\frac{H^{N}(\vec{P})+e_{H^{e}}(\vec{q}, \vec{p}, \vec{R})+\frac{p_{s}^{2}}{2 Q}-E}{g k T}\right),
$$

and write

$Z=\int_{M_{C} \times M_{Q} \times \mathbb{R}^{2}} \mathrm{~d} \mu_{C} \mathrm{~d} \mu_{Q} \mathrm{~d} p_{S} \mathrm{~d} s s^{D} \delta\left[s-\exp \left(-\frac{H^{N}(\vec{P})+e_{H^{e}}(\vec{q}, \vec{p}, \vec{R})+\frac{p_{s}^{2}}{2 Q}-E}{g k T}\right)\right]$.

Integrating in the variable $s$, we obtain

$$
\begin{aligned}
Z=\frac{1}{g k T} \int \mathrm{d} p_{s} & \exp \left[\frac{D+1}{g k T}\left(E-\frac{p_{s}^{2}}{2 Q}\right)\right] \\
& \times \int_{M_{C} \times M_{Q}} \mathrm{~d} \mu_{C} \mathrm{~d} \mu_{Q} \exp \left[-\frac{D+1}{g k T}\left(H^{N}(\vec{P})+e_{H^{e}}(\vec{q}, \vec{p}, \vec{R})\right] .\right.
\end{aligned}
$$


Then, if we choose $g=D+1$, the exponential takes the desired form. Besides, we can note that when calculating the average of any function $M \in \mathcal{O}$ (where $\mathcal{O}$ corresponds to equation (43) and therefore $M$ does not depend on the extended variables), the quantity

$$
\frac{1}{(D+1) k T} \int \mathrm{d} p_{s} \exp \left[\frac{1}{k T}\left(E-\frac{p_{s}^{2}}{2 Q}\right)\right]
$$

factorizes. If we also assume that the function $M$ does not depend either on the norm of the quantum state (as it is the case for the quantum observables) and we decompose the volume element $\mathrm{d} \mu_{Q}$ as equation (58), the radial part of the quantum integral also factorizes and cancels the analogous term arising from the partition function.

Thus, we can write

$$
\begin{aligned}
& \langle M\rangle=1 / Z \int_{M_{C} \times M_{Q} \times \mathbb{R}^{2}} \mathrm{~d} \mu_{C} \mathrm{~d} f_{\mathbb{I}} \mathrm{d} \tilde{\Omega}_{Q} \mathrm{~d} p_{s} \mathrm{~d} s M(\vec{R}, \vec{P}, \vec{q}, \vec{p}) \delta\left(H\left(\vec{R}, \vec{P}, \vec{q}, \vec{p}, s, p_{s}\right)-E\right) \\
& =1 / Z\left(\frac{1}{(D+1) k T} \int \mathrm{d} p_{s} \exp \left[\frac{1}{k T}\left(E-\frac{p_{s}^{2}}{2 Q}\right)\right] \int_{\mathbb{R}} \mathrm{d} f_{\mathbb{I}}\right. \\
& \left.\times \int_{M_{C} \times S_{Q}} \mathrm{~d} \mu_{C} \mathrm{~d} \Omega_{Q} M(\vec{R}, \vec{P}, \vec{q}, \vec{p}) \exp \left[-\frac{1}{k T}\left(H^{N}(\vec{P})+e_{H^{e}}(\vec{q}, \vec{p}, \vec{R})\right)\right]\right),
\end{aligned}
$$

where

$$
\begin{aligned}
Z=\frac{1}{(D+1) k T} & \int \mathrm{d} p_{s} \exp \left[\frac{1}{k T}\left(E-\frac{p_{s}^{2}}{2 Q}\right)\right] \int_{\mathbb{R}} \mathrm{d} f_{\mathbb{I}} \\
& \times \int_{M_{C} \times S_{Q}} \mathrm{~d} \mu_{C} \mathrm{~d} \Omega_{Q} \exp \left[-\frac{1}{k T}\left(H^{N}(\vec{P})+e_{H^{e}}(\vec{q}, \vec{p}, \vec{R})\right)\right] .
\end{aligned}
$$

Hence, we have proved that the microcanonical distribution of the extended dynamical system created by equation (69) is equivalent to the canonical distribution created by the Hamiltonian

$$
H(\vec{R}, \vec{P}, \vec{q}, \vec{p})=H^{N}(\vec{P})+e_{H^{e}}(\vec{q}, \vec{p}, \vec{R})
$$

\section{Conclusions and future work}

In this paper, we have constructed a rigorous Hamiltonian description of the Ehrenfest dynamics of an isolated system by combining the Poisson bracket formulation of classical mechanics with the geometric formulation of quantum mechanics. We have also constructed the corresponding statistical description and obtained the associated Liouville equation. Finally, after verifying numerically that the Ehrenfest dynamics is ergodic, we justify the equilibrium distribution produced by it.

It is important to keep in mind the restriction to finite-dimensional quantum systems that we fixed as starting point, since our first goal is to apply our construction to molecular dynamics. From a theoretical point of view, though, it is very interesting to perform the same type of analysis for infinite-dimensional quantum systems (note that the Ehrenfest dynamics as formulated in $[4,5]$ consists of classical nuclei and an infinite-dimensional Hilbert space for the electronic subsystem). Formally, the construction can be defined in the same way, once we adapt the language to infinite-dimensional manifolds. But the nonlinearity of the resulting dynamics imposes very challenging problems at many levels, for instance at the very existence of the solutions of the Hamiltonian dynamical flow. Another interesting problem would be to recover, within the geometrical formalism, the classical-quantum system as a suitable classical 
limit for the nuclear degrees of freedom of an original completely quantum system (similarly to what is done in $[4,5]$ in the standard approach). We hope to address these problems in the future.

The definition of the Hamiltonian in equation (49) and its associated canonical equilibrium distribution (equation (67)) allows us now to use (classical) Monte Carlo methods for computing canonical equilibrium averages in our system, given by expression (56). But this formalism also allows us to easily introduce a Nosé extended system scheme to compute averages in the canonical ensemble by performing constant energy molecular dynamics simulations. This is a very important feature of the final setting, because the situations where the Ehrenfest approach is necessary (i.e. those where the electronic energy gap is small and the Born-Oppenheimer approximation is not sufficient) the effect of the temperature can be relevant. A method for the simulation of the canonical ensemble is thus of greatest importance.

Alternatively, if we wish to perform MD simulations using stochastic methods, we should first construct the Langevin dynamics associated with our Hamiltonian (49) and equations (50)-(53). To develop this program, we also need an analogue of the FokkerPlanck equation associated with those Langevin equations and then we have to check whether its solution at infinite time approaches equation (67). Presently, we are working on this point [50], i.e. on the extension of our formalism to an associated stochastic MD.

\section{Acknowledgments}

We would like to thank José F Cariñena, Andrés Cruz and David Zueco for many illuminating discussions. This work has been supported by the research projects E24/1, E24/2 and E24/3 (DGA, Spain), FPA2009-09638 (CICYT, Spain), FIS2009-13364-C02-01 (MICINN, Spain), 200980 I064 (CSIC, Spain) and ARAID and Ibercaja grant for young researchers (Spain). We also acknowledge the suggestions and insights of the referees.

\section{References}

[1] Marx D and Hutter J 2009 Ab Initio Molecular Dynamics: Basic Theory and Advanced Methods (Cambridge: Cambridge University Press)

[2] Gerber R B, Buch V and Ratner M A 1982 Time-dependent self-consistent field approximation for intramolecular energy transfer: I. Formulation and application to dissociation of van der Waals molecules J. Chem. Phys. 77 3022-30

[3] Gerber R B and Ratner M A 1988 Self-consistent field methods for vibrational excitations in polyatomic systems Advances in Chemical Physics 70 97-132

[4] Bornemann F A, Nettesheim P and Schütte C 1996 Quantum-classical molecular dynamics as an approximation to full quantum dynamics J. Chem. Phys. 105 1074-83

[5] Bornemann F A, Nettesheim P and Schütte C 1995 Quantum-classical molecular dynamics as an approximation to full quantum dynamics Technical Report SC-95-26, Konrad-Zuse-Zentrum

[6] Zhu C, Jasper A W and Truhlar D G 2005 Non-Born-Oppenheimer Liouville-von Neumann dynamics: evolution of a subsystem controlled by linear and population-driven decay of mixing with decoherent and coherent switching J. Chem. Theor. Comput. 1 527-40

[7] Heslot A 1985 Quantum mechanics as a classical theory Phys. Rev. D 31 1341-8

[8] Parandekar P V and Tully J C 2006 Detailed balance in Ehrenfest mixed quantum-classical dynamics J. Chem. Theor. Comput. 2 229-35

[9] Käb G 2006 Fewest switches adiabatic surface hopping as applied to vibrational energy relaxation J. Phys. Chem. A 110 3197-215

[10] Bastida A, Cruz C, Zúñiga J, Requena A and Miguel B 2006 A modified Ehrenfest method that achieves Boltzmann quantum state populations Chem. Phys. Lett. 417 53-7

[11] Käb G 2002 Mean field Ehrenfest quantum/classical simulation of vibrational energy relaxation in a simple liquid Phys. Rev. E 66046117 
[12] Tully J C 1998 Nonadiabatic Dynamics (Singapore: World Scientific) pp 34-72

[13] Müller U and Stock G 1997 Surface-hopping modeling of photoinduced relaxation dynamics on coupled potential-energy surfaces J. Chem. Phys. 107 6230-45

[14] Mauri F, Car R and Tosatti E 1993 Canonical statistical averages of coupled quantum-classical systems Europhys. Lett. 24 431-6

[15] Terashima T, Shiga M and Okazaki S 2001 A mixed quantum-classical molecular dynamics study of vibrational relaxation of a molecule in solution J. Chem. Phys. 114 5663-73

[16] Alonso J L, Castro A, Echenique P, Polo V, Rubio A and Zueco D 2010 Ab initio molecular dynamics on the electronic Boltzmann equilibrium distribution New J. Phys. 12083064

[17] Nosé S 1984 A unified formulation of constant temperature molecular dynamics methods J. Chem. Phys. 81 511-9

[18] Nosé S 1991 Constant temperature molecular dynamics methods Prog. Theor. Phys. Suppl. 103 1-46

[19] Kisil V V 1996 No more than mechanics: I J. Nat. Geom. 9 1-14 (arXiv:functan/9405002v3)

[20] Prezhdo O V and Kisil V V 1997 Mixing quantum and classical mechanics Phys. Rev. A 56 162-175

[21] Kapral R and Ciccotti G 1999 Mixed quantum-classical dynamics J. Chem. Phys. 110 8919-29

[22] Nielsen S, Kapral R and Ciccotti G 2001 Statistical mechanics of quantum-classical systems J. Chem. Phys. 115 5805-15

[23] Kapral R 2001 Quantum-classical dynamics in a classical bath J. Phys. Chem A 105 2885-9

[24] Kisil V V 2005 A quantum-classical bracket from p-mechanics Europhys. Lett. 72 873-9

[25] Agostini F, Caprara S and Ciccotti G 2007 Do we have a consistent non-adiabatic quantum-classical mechanics? Europhys. Lett. $\mathbf{7 8} 30001$

[26] Kisil V V 2010 Comment on 'Do we have a consistent non-adiabatic quantum-classical mechanics?' by Agostini F. et al. Europhys. Lett. 8950005

[27] Agostini F, Caprara S and Ciccotti G 2010 Reply to the comment by V V Kisil Europhys. Lett. 8950006

[28] Schmitt U and Brickmann J 1996 Discrete time-reversible propagation scheme for mixed quantum-classical dynamics Chem. Phys. 208 45-56

[29] Kibble T W B 1979 Geometrization of quantum mechanics Commun. Math. Phys. 65 189-201

[30] Abbati M C, Cirelli R, Lanzavecchia P and Mania A 1984 Pure states of general quantum-mechanical systems as Kähler bundles Nuovo Cimento B 83 43-60

[31] Cirelli R, Manià A and Pizzocchero L 1991 Quantum phase space formulation of Schrödinger mechanics Int. J. Mod. Phys. A $62133-46$

[32] Brody D C and Hughston L P 2001 Geometric quantum mechanics J. Geom. Phys. 38 19-53

[33] Ashtekar A and Schilling T A 1998 Geometrical Formulation of Quantum Mechanics On Einstein Path: Essays in Honor of Englebert Schucking ed A Harvey (Berlin: Springer) pp 23-65

[34] Cariñena J F, Clemente-Gallardo J and Marmo G 2006 Introduction to quantum mechanics and the quantumclassical transition Proc. 15th Int. Workshop on Geometry and Physics (RSME) vol 11 pp 3-45

[35] Cariñena J F, Clemente-Gallardo J and Marmo G 2007 Geometrization of quantum mechanics Theor. Math. Phys. 152 894-903

[36] Clemente-Gallardo J and Marmo G 2008 Basics of quantum mechanics, geometrization and some applications to quantum information Int. J. Geom. Methods Mod. Phys. 5 989-1032

[37] Balescu R 1997 Statistical Dynamics: Matter Out of Equilibrium (London: Imperial College Press)

[38] Balescu R 1975 Equilibrium and Nonequilibrium Statistical Mechanics (New York: Wiley-Interscience)

[39] Tolman R C 1938 The Principles of Statistical Mechanics (Oxford: Clarendon)

[40] Andrade X, Castro A, Zueco D, Alonso J L, Echenique P, Falceto F and Rubio A 2009 Modified Ehrenfest formalism for efficient large-scale ab initio molecular dynamics J. Chem. Theor. Comput. 5 728-42

[41] Abraham R and Marsden J E 1978 Foundations of Mechanics (Reading, MA: Addison Wesley)

[42] Landsman N P 1998 Mathematical Topics Between Classical and Quantum Mechanics (Berlin: Springer)

[43] Oliveira C R and Werlang T 2007 Ergodic hypothesis in classical statistical mechanics Revista Brasileira de Ensino de Física 29 189-201

[44] Schwabl F 2002 Statistical Mechanics (Berlin: Springer)

[45] Jaynes E T 1957 Information theory and statistical mechanics I Phys. Rev. 106 620-30

[46] Ellis R S 1985 Entropy, Large Deviations and Statistical Mechanics (Berlin: Springer)

[47] Touchette H 2009 The large deviation approach to statistical mechanics Phys. Rep. 478 1-69

[48] Reimann P 2008 Foundation of statistical mechanics under experimentally realistic conditions Phys. Rev. Lett. 101190403

[49] Reimann P 2010 Canonical thermalization New J. Phys. 12055027

[50] Alonso J L, Castro A, Clemente-Gallardo J, Cuchí J C, Echenique P, Falceto F and Zueco D 2011 in preparation 